\title{
Numerical verification of an analytical model for phase noise in MEMS oscillators
}

\author{
D. K. Agrawal, F. Bizzarri, Senior Member, IEEE, A. Brambilla, A. A. Seshia, Senior Member, IEEE
}

\begin{abstract}
A new analytical formulation for phase noise in MEMS oscillators was recently presented encompassing the role of essential non-linearities in the electrical and mechanical domains. In this paper we validate the effectiveness of the proposed analytical formulation with respect to the unified theory developed by Demir et al. describing phase noise in oscillators. In particular, it is shown that, over a range of the second order mechanical nonlinear stiffness of the MEMS resonator, both models exhibit an excellent match in the phase diffusion coefficient calculation for a square-wave MEMS oscillator.
\end{abstract}

Index Terms-Microelectromechanical systems, oscillator, phase noise, hybrid system, saltation matrix.

\section{INTRODUCTION}

There has been much recent interest in the analysis of noise in MEMS oscillators and in particular, towards developing models providing analytical insight into the role of non-linear effects on the frequency and phase noise. The availability of such a model is cardinal since, in general, they enable designer insight into the underlying physics and provide a starting basis for more detailed design optimization studies. Thus, it is worth realizing that either the numerical or the experimental validation of the effectiveness of a given analytical model is crucial, together with a proper knowledge of its limits. Recently, an analytical formulation providing physical insight into the role of non-linear effects on phase noise in MEMS oscillators was proposed [1] with model predictions shown to be qualitatively consistent with experimental findings in the literature. This paper addresses an independent validation of this model by comparing the findings with a previously established approach for phase noise analysis in non-linear oscillators [2]. It is worth mentioning that the approach developed in [2] has been extended in [3] in which a more general theory for the nonlinear perturbative analysis of noise in free running oscillators affected by white Gaussian noise sources was presented, able to include the effects of phase noise, orbital fluctuations and their correlation. Nevertheless, in this work a direct comparison with [2] is chosen since in their work Demir et al. proposed a unifying theory to characterize noise in oscillators which leads to the calculation of $c$, a single

D. K. Agrawal and A. A. Seshia are with Department of Engineering, University of Cambridge, UK. \{dka23, aas41\}@cam.ac.uk

F. Bizzarri, A. Brambilla are with Politecnico di Milano, Dipartimento di Elettronica e Informazione, p.za. Leonardo da Vinci, n. 32, I20133 Milano, Italy. \{federico.bizzarri, angelo.brambilla\} @ polimi.it

D. K. Agrawal is also with Department of Chemical and Biomolecular Engineering, Johns Hopkins University, USA. dagrawa5@jhu.edu

F. Bizzarri is also with the Advanced Research Center on Electronic Systems for Information and Communication Technologies E. De Castro (ARCES), University of Bologna, Italy. scalar quantity representing the variance of the per-cycle jitter and the spectral spreading in a noisy oscillator [4]. The $c$ constant can be evaluated from the left $v_{1}(t)$ eigenfuntion (referred to as PPV in the literature [4]) of the fundamental matrix of the noiseless oscillator [5]. This eigenfunction is associated to the unit multiplier of the monodromy matrix and must be evaluated in one working period. Beside numerical methods and technicalities involved in the evaluation of $c$, from a theoretical point of view the linearisation of the noiseless oscillator dynamics along its steady state periodic solution must exist. If this is not the case, the fundamental matrix and hence $v_{1}(t)$ is not defined. Consequently, oscillators described by a non smooth vector field (as it happens for analog mixed signal (AMS) circuits) must be handled with care. Bizzarri et al. have shown and experimentally verified [6]-[9] that, by resorting to the saltation matrix linear operator [10], the $c$ constant can be evaluated also for AMS oscillators, that belong to the wider class of hybrid dynamical systems. In general, the saltation matrix can be viewed as a correction factor to be properly inserted at those points where the fundamental matrix is not defined. It is worth noticing that such critical points are those where the noiseless orbit is not differentiable because of the discontinuity of the vector field.

In this paper, a case study of an electrostatically addressed MEMS resonator-based square-wave oscillator, in which the resonator and oscillator circuit nonlinearities are integrated into a single modeling framework, is investigated. Noise sources in the oscillator are viewed as an equivalent noise voltage source representative of a wide-sense stationary process such as white noise. After demonstrating that the derived single-sideband phase noise PSD around the first harmonic in [1] can be reduced to (41) in [2], the counterpart of the $c$ constant in such an expression is identified as a function of the proposed model parameters. Finally, the numerically computed constant $c$ is compared with the values determined using the analytically derived expression of $D_{\phi_{1}}$ and a very good agreement is demonstrated. The results presented in this work are based on two first order differential equations with discontinuous right-hand side. For this reason, a recent extension [6] of Demir's approach to hybrid dynamical systems is considered. Finally, the numerical validation of the analytical noise model proposed in [1] for the considered case study, also suggests validity limits of the model itself with respect to the second order mechanical nonlinear stiffness of the MEMS resonator. 


\section{MODEL DESCRIPTION}

An oscillator can be conceptually represented by three elements placed in a closed-loop: a resonator, a gain element and an amplitude-limiting mechanism necessary to regulate oscillation amplitude. ${ }^{1}$ The phase noise modeling approach introduced in [1] is devoted to a wide class of oscillators enclosing an electrostatically driven MEMS resonator as a frequencyselective element providing a low-loss non-linear second-order response. In particular, we consider an architecture made up of an electrostatically driven double-ended-turning fork (DETF) silicon micro-resonator, a front-end transresistance amplifier, a bandpass filter and a comparator as illustrated in Fig. 1 [11].

A simplified description of an oscillator belonging to such a class, driven by random noise excitation ( $v_{n}$ in Eq. (1)), is obtained in terms of a second-order nonlinear stochastic differential equation as [1], [12]

$$
\begin{aligned}
\frac{d^{2}}{d t^{2}} v(t)+\alpha \frac{d}{d t} v(t)-\beta \operatorname{sign}\left(\frac{d}{d t} v(t)\right)+ & \omega_{0}^{2} v(t)+\mu v^{3}(t) \\
& =-\frac{R_{f}}{L_{m}} \omega_{o} v_{n},
\end{aligned}
$$

where

$$
\alpha=\frac{R_{m}}{L_{m}}, \beta=\frac{R_{f}}{L_{m}} \frac{\pi \omega_{o} V_{F}}{4}, \mu=\frac{1}{R_{f}^{2} L_{m} C_{m 2}} .
$$

Here, $L_{m}, R_{m}$ and $C_{m o}$ are the equivalent motional inductance, resistance and capacitance respectively while $Q$ is the quality factor of the operating vibration mode and $V_{F}$ is the output amplitude of the hard limiter at the oscillation frequency $\left(\omega_{o}\right)$ which may be expressed as $1 / \sqrt{L_{m} C_{m o}} . R_{f}$ is the feedback resistance of the transimpedance amplifier while $C_{m 2}$ is governed by the cubic nonlinearity of the resonator and is indirectly proportional to the summation of the second order nonlinear electrical $\left(k_{2 e}\right)$ and mechanical $\left(k_{2 m}\right)$ stiffness of the resonator [12]. Moreover, $\operatorname{sign}(y)=1$ if $y \geq 0$ and $\operatorname{sign}(y)=-1$ otherwise. $^{2}$ Considering $25 \mathrm{~V}$ and $30 \mathrm{~V}$ $V_{d c}$ and a set of excitation voltages, system parameters in Eq. (1) are determined from the measured open-loop response

\footnotetext{
${ }^{1}$ This amplitude-limited mechanism may be engineered to provide a gain roll-off at large amplitudes or may be inherent to the nonlinearities that are operative in the resonator.

${ }^{2}$ Note that if one assumes $\operatorname{sign}(0)=0$, Eq. (1) exhibits a stable equilibrium point on the manifold $\frac{d v}{d t}=0$ ruling the switching of the vector field.
}

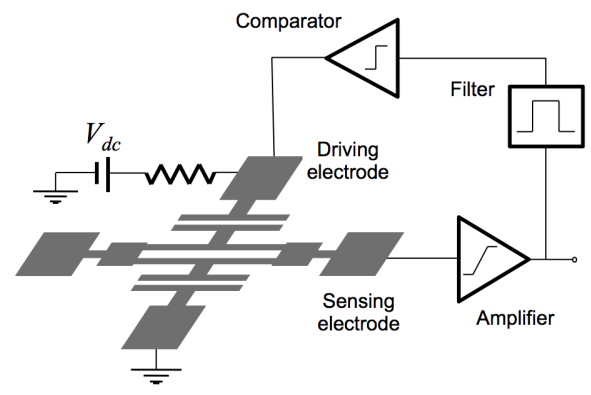

Figure 1. Schematic description of the MEMS square wave oscillator. of the resonator. The calculated values of these parameters for two different $V_{d c}$ voltages are shown in Table II [1]. Using these values, for different excitation voltages $\left(V_{F}\right) \beta$ can be determined as shown in Table II.

The phase noise model derived in [1] explains the underlying phenomenon by which inherent noise in the oscillator results in perturbations in the phase trajectory. This is achieved introducing the $D_{\phi_{1}}$ diffusion coefficient. The rate at which state point in the phase plane $(v, \dot{v})$ diffuses turns out to be directly proportional to $D_{\phi_{1}}$ which is expressed as

$$
D_{\phi_{1}}=D_{\phi_{o}}\left(1+\gamma^{2}\right)
$$

where

$$
\begin{aligned}
& D_{\phi_{o}}=\frac{R_{m}^{2}}{L_{m}^{2} V_{F}^{2}} \frac{\bar{v}_{n}^{2}}{4} \\
& \gamma=\frac{3 V_{F}^{2}}{2 \omega_{o} C_{m 2} R_{m}^{3}}
\end{aligned}
$$

and $\overline{v_{n}^{2}}$ is the singled-sided PSD of the equivalent noise voltage source in Eq. (1).

According to the analysis conducted in [1], assuming that the actual angular frequency $\omega$ of the oscillator can be safely approximated by $\omega_{o}$, a straightforward relation exists between $D_{\phi_{1}}$ and the Demir's constant $c$,

$$
c=\frac{D_{\Phi_{1}}}{\omega^{2}} .
$$

This relation can be derived by a direct comparison between the expressions of the single-sideband phase noise spectrum around the oscillator fundamental frequency, derived in [1] (Eq. (81)) and [2] (Eq. (41)).

For each value of $V_{d c}$ and $V_{F}$, the diffusion coefficient $\left(D_{\Phi_{1}}\right)$ can be calculated using the analytical expression shown in (3-5) [1]. The results are shown in Table III

Table I

SUMMARY OF THE CALCULATED PARAMETERS OF THE DETF MEMS RESONATOR.

\begin{tabular}{lcc}
\hline Parameter & Value at $25 \mathrm{~V} V_{\mathrm{dc}}$ & Value at $30 \mathrm{~V} V_{\mathrm{dc}}$ \\
\hline \hline$L_{m}(\mathrm{H})$ & $8.4 \times 10^{3}$ & $5.8 \times 10^{3}$ \\
$R_{m}(\Omega)$ & $8.1 \times 10^{5}$ & $7 \times 10^{5}$ \\
$C_{m o}(\mathrm{~F})$ & $6 \times 10^{-17}$ & $8.6 \times 10^{-17}$ \\
$C_{m 2}\left(\mathrm{FA}^{2}\right)$ & $1.2 \times 10^{-27}$ & $2.7 \times 10^{-27}$ \\
\hline
\end{tabular}

Table II

Calculated values of $\beta$ AT Different $V_{d c}$ ANd $V_{F}$ voltages.

\begin{tabular}{lcc}
\hline$V_{F}(\mathrm{mV})$ & $\beta$ at $25 \mathrm{~V} V_{d c}$ & $\beta$ at $30 \mathrm{~V} V_{d c}$ \\
\hline \hline 16 & $7.046907072834351 \cdot 10^{3}$ & $10.1475461848815 \cdot 10^{3}$ \\
32 & $1.406042811951403 \cdot 10^{4}$ & $2.02470164921002 \cdot 10^{4}$ \\
50 & $2.228427680970662 \cdot 10^{4}$ & $3.20893586059775 \cdot 10^{4}$ \\
100 & $4.446297773474253 \cdot 10^{4}$ & $6.40266879380292 \cdot 10^{4}$ \\
160 & $7.046907072834347 \cdot 10^{4}$ & $10.1475461848815 \cdot 10^{4}$ \\
\hline
\end{tabular}


Table III

ANALYTiCAlly CALCUlated VALUE OF $D_{\Phi_{1}}$ AT DifFERENT $V_{d c}$ AND $V_{F}$ VOLTAGES [1]

\begin{tabular}{lcc}
\hline$V_{F}(\mathrm{mV})$ & $D_{\Phi_{1}}$ at $25 \mathrm{~V} V_{d c}$ & $D_{\Phi_{1}}$ at $30 \mathrm{~V} V_{d c}$ \\
\hline \hline 16 & $1.841659481347613 \cdot 10^{-8}$ & $2.62265679278919 \cdot 10^{-8}$ \\
32 & $1.531718125668003 \cdot 10^{-8}$ & $1.39692408865264 \cdot 10^{-8}$ \\
50 & $3.021850186234356 \cdot 10^{-8}$ & $2.22147204131426 \cdot 10^{-8}$ \\
100 & $1.145019624210766 \cdot 10^{-7}$ & $7.93943342206827 \cdot 10^{-8}$ \\
160 & $2.867902739961938 \cdot 10^{-7}$ & $1.98142108244848 \cdot 10^{-7}$ \\
\hline
\end{tabular}

\section{NUMERICAL Simulation}

To validate the analytical model summarized in Section II it is necessary, for several sets of parameters values, (i) to derive the $c$ values of the dynamical system described by Eq. (1) directly from Demir's approach, (ii) compute $D_{\phi_{1}}$ according to Eq. (3), (iii) compute $c$ through Eq. (6) and compare these values with those obtained at point (i). We start by rewriting Eq. (1) as

$$
\left\{\begin{array}{l}
\dot{x}_{1}(t)=\underbrace{x_{2}(t)}_{f_{1}\left(x_{1}, x_{2}\right)} \\
\dot{x}_{2}(t)=\underbrace{-\alpha x_{2}(t)+\beta \operatorname{sign}\left(x_{2}(t)\right)-\omega_{0}^{2} x_{1}(t)-\mu x_{1}^{3}(t)}_{f_{2}\left(x_{1}, x_{2}\right)}
\end{array},\right.
$$

where $x_{1}(t)=v(t)$ and $x_{2}(t)=\dot{v}(t)$ and the $v_{n}$ noise source is dropped.

Equation (7) represents a hybrid dynamical system [10] since its vector field exhibits discontinuities (switching) whenever the trajectory in the state space reaches the manifold $h\left(x_{1}, x_{2}\right)=x_{2}=0$. For our purposes the main consequence of this phenomenon is that the variational problem associated to (7) is not defined at the discontinuity boundary identified by the aforementioned manifold. For this reason one must resort to the saltation matrix linear operator [10] in order to evaluate the correct fundamental matrix solving the variational problem. This matrix is crucial since it allows (i) to derive efficiently the steady state periodic solution of Eq. (7) through a time-domain shooting method, (ii) to study the stability of this solution through its Floquet multipliers, (iii) to derive the PPV $v_{1}(t)$ (the key ingredient to obtain $c$ ).

As an example, for $V_{d c}=30 \mathrm{~V}$ and $V_{F}=16 \mathrm{mV}$, the angular frequency of the steady state periodic solution is $\omega=1.4156 \cdot 10^{6} \mathrm{rads}^{-1}$ and its numerical computed Floquet multipliers are $\lambda_{1}=1.0000$ (this is the theoretical unit multiplier) and $\lambda_{2}=0.9997$. Since $\lambda_{2}$ stays within the unit circle in the complex plane, the periodic solution is stable. Moreover, as it can be easily verify by performing a transient simulation of system (7), since $\lambda_{2}$ is very close to 1 the considered oscillator exhibits a relaitvely high $Q$ factor.

Once the noiseless periodic state solution is available, together with the evolution of the system fundamental matrix along one working period $T=2 \pi / \omega$ of the oscillator, it is now possible to compute the $c$ constant. As a first step, the left eigenfuntion $v_{1}(t)$ of the fundamental matrix must be computed. It has been obtained by resorting to the MATLAB $^{\mathrm{TM}}$ version of the suite SUNDIALS [13], performing the backward integration from $T$ to 0 of the adjoint system corresponding to Eq. (7). As an initial condition for the backward integration, the eigenvector corresponding to the unitary eigenvalue of the monodromy matrix has been chosen.

As a second step, the $B\left(x_{1}, x_{2}\right)$ (incidence) matrix describing how noise sources propagate must be provided. In particular a noisy version of Eq. (7) must be written as

$$
\frac{d}{d t}\left[\begin{array}{l}
x_{1}(t) \\
x_{2}(t)
\end{array}\right]=\left[\begin{array}{l}
f_{1}\left(x_{1}, x_{2}\right) \\
f_{2}\left(x_{1}, x_{2}\right)
\end{array}\right]+B\left(x_{1}, x_{2}\right) \eta(t)
$$

where $\eta(t)$ is a $p$-dimensional vector of uncorrelated white noise sources and $B\left(x_{1}, x_{2}\right)$ is a $2 \times p$ matrix. In this case, $p=1$ and $B\left(x_{1}, x_{2}\right)=(0, \sqrt{\zeta})^{\mathrm{T}}$ is a constant vector. Hence, according to [2],

$$
c=\frac{\zeta}{T} \int_{T} v_{1}^{\mathrm{T}}(t) v_{1}(t) d t
$$

Moreover, using (2) and (8), we derived an expression that relate $\zeta$ with the parameters of the oscillator as

$$
\sqrt{\zeta}=\frac{R_{f}}{L_{m}} \omega_{o} \frac{\overline{v_{n}^{2}}}{2}
$$

Note that the variance of the per cycle time $c$ and phase jitter $D_{\Phi_{1}}$ are related by the expression mentioned in Eq. (6). It is therefore now possible, for any set of parameters characterizing Eq. (1), to compare the $c$ value obtained by resorting to Eq. (9) and the one coming from Eq. (6). A particular set of results is shown in Fig. 2 for $k_{2 m}=1.43 \cdot 10^{12} \mathrm{Nm}^{3}$, which corresponds to a particular value of the second order mechanical nonlinear stiffness of the MEMS resonator previously reported in [12]. These results are obtained for two different values of $V_{d c}$ voltage with a subset of $V_{F}$ voltages. This allows to test both the parameter sets reported in Table II. A good agreement can be observed thus validating the analytical phase noise model under these conditions. As predicted by the analytical model in [12], an optimal operating point for the oscillator (particular value of $V_{F}$ ) exists where the phase noise of the oscillator is minimized.

A second set of results are obtained for $V_{d c}=30 \mathrm{~V}$ and scaling the $k_{2 m}$ value previously adopted by a factor $\kappa$. A good agreement between the analytical and numerical predictions is generally seen and the results are consistent with the trends observed in Fig. 3. It is worth noticing that a particular value of $k_{2 m}(\kappa=0.27)$ exists such that a best case noise performance is observed which can further be improved by increasing the feedback signal $V_{F}$. This happens when the second order mechanical and electrical stiffness corrections cancel each other and lead to an expression of $D_{\phi_{1}}$ which is independent of $\gamma$. This behavior is consistent with the previously reported experimental observations of phase noise optimization in MEMS oscillators [14]. As the value of $k_{2 m}$ is then progressively increased, we note that the optimal operating point of the oscillator shifts to lower feedback voltages as predicted by the analytical model. This can be inferred from Eq. (3- 5). An increase in $k_{2 m}$, increases the contribution of the resonator cubic nonlinearities to the diffusion coefficient which can be reduced by lowering the feedback voltage. This validation provides further weight to the design insight gained by the analytical formulation, particularly with regard to the 


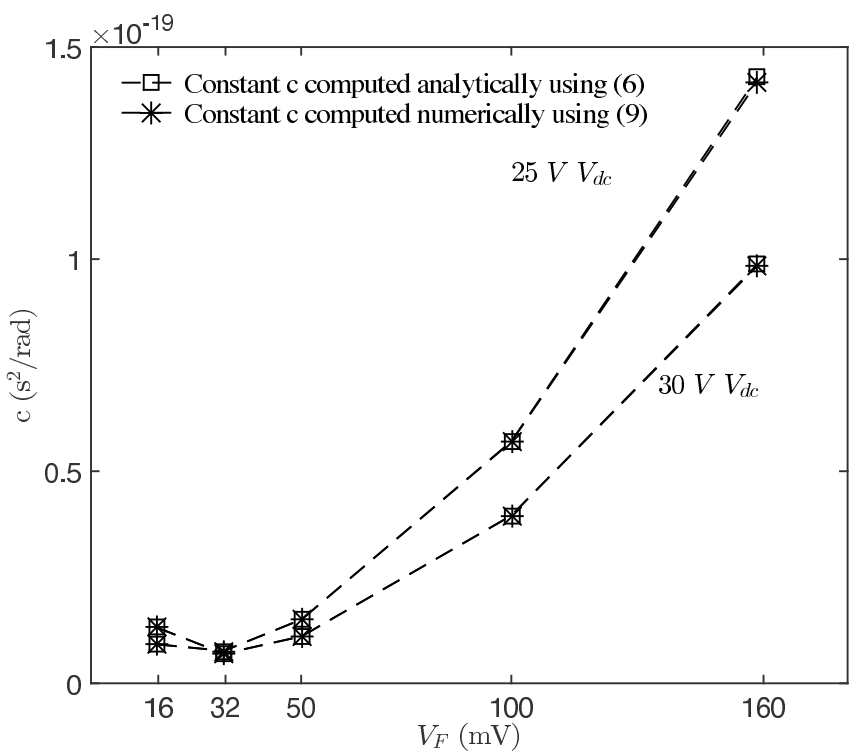

Figure 2. A comparison between the phase noise calculations proposed in [2] and [1] is carried out by comparing the $c$ values calculated using the two approaches.

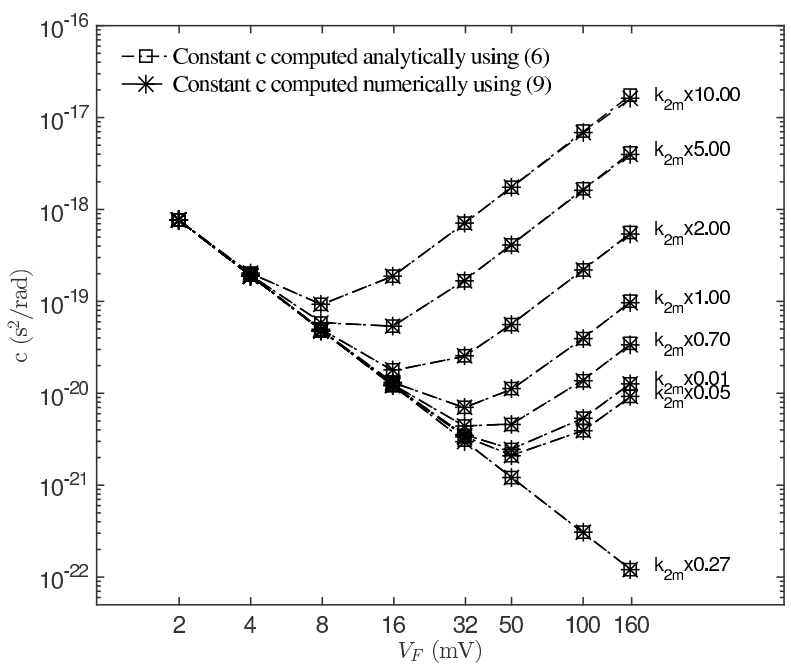

Figure 3. Here, the second order mechanical nonlinear stiffness of the resonator is varied by multiplying a known constant value.

role of resonator and amplifier non-linearity in determining the oscillator phase noise.

\section{CONCLUDING REMARKS}

This paper reports the numerical verification of a recently proposed analytical model for phase noise in a MEMS oscillator embedding a non-linear resonator. The effectiveness of the proposed analytical formulation is proven with respect to the unified theory developed by Demir et al. describing phase noise in oscillators. In particular, it is shown that, over a range of values for the second order mechanical nonlinear stiffness of the MEMS resonator, both models exhibit an excellent match in the phase diffusion coefficient calculation for a square-wave
MEMS oscillator. The numerical model verifies the trends described by the analytical model with respect to the trends in phase noise for values of resonator polarization voltage and oscillator feedback voltage. It is also seen that for a particular value of non-linear stiffness parameter $k_{2 m}$, there exists an optimal operating point in the feedback configuration where the phase noise of the oscillator is minimized. Furthermore, by setting the values of the non-linear electrical spring $k_{2 e}$ to equal the non-linear mechanical spring $k_{2 m}$, it is possible to improve the oscillator noise performance, consistent with independently reported experimental observations to this effect.

\section{REFERENCES}

[1] D. K. Agrawal and A. A. Seshia, "An analytical formulation for phase noise in mems oscillators," IEEE Transactions on Ultrasonics, Ferroelectrics and Frequency Control, vol. 61, no. 12, pp. 1938-52, 2014.

[2] A. Demir, A. Mehrotra, and J. Roychowdhury, "Phase noise in oscillators: a unifying theory and numerical methods for characterization," Circuits and Systems I: Fundamental Theory and Applications, IEEE Transactions on, vol. 47, no. 5, pp. 655-674, May 2000.

[3] F. L. Traversa and F. Bonani, "Oscillator noise: a nonlinear perturbative theory including orbital fluctuations and phase-orbital correlation," Circuits and Systems I: Regular Papers, IEEE Transactions on, vol. 58, no. 10, pp. 2485-2497, 2011.

[4] A. Demir and J. Roychowdhury, "A reliable and efficient procedure for oscillator ppv computation, with phase noise macromodeling applications," IEEE Transactions on Computer-Aided Design of Integrated Circuits and Systems, vol. 22, pp. 188-197, Feb. 2003.

[5] M. Farkas, Periodic motions. New York, NY, USA: Springer-Verlag New York, Inc., 1994.

[6] F. Bizzarri, A. Brambilla, and G. Storti Gajani, "Steady state computation and noise analysis of analog mixed signal circuits," Circuits and Systems I: Regular Papers, IEEE Transactions on, vol. 59, no. 3, pp. 541 -554, march 2012.

[7] — "Phase noise simulation in analog mixed signal circuits: An application to pulse energy oscillators," Circuits and Systems II: Express Briefs, IEEE Transactions on, vol. 58, no. 3, pp. 154 -158, March 2011.

[8] — , "Extension of the variational equation to analog/digital circuits: numerical and experimental validation," International Journal of Circuit Theory and Applications, vol. 41, no. 7, pp. 743-752, 2013.

[9] F. Bizzarri, A. Brambilla, G. Storti Gajani, and S. Banerjee, "Simulation of real world circuits: Extending conventional analysis methods to circuits described by heterogeneous languages," IEEE Circuits and Systems Magazine, vol. 14, no. 4, pp. 51-70, 2014.

[10] M. Di Bernardo, C. Budd, A. Champneys, and P. Kowalczyk, Piecewisesmooth Dynamical Systems, Theory and Applications. London: Springer-Verlag, 2008.

[11] J. E. Y. Lee, B. Bahreyni, Y. Zhu, and A. A. Seshia, "A single-crystalsilicon bulk-acoustic-mode microresonator oscillator," IEEE Electron Device Letters, vol. 29, no. 7, pp. 701-703, 2008.

[12] D. K. Agrawal, J. Woodhouse, and A. A. Seshia, "Modelling nonlinearities in mems oscillators," IEEE Transactions on Ultrasonics, Ferroelectrics and Frequency Control, vol. 60, no. 8, pp. 1646-1659, 2013.

[13] A. Hindmarsh, P. Brown, K. Grant, S. Lee, R. Serban, D. Shumaker, and C. Woodward, "Sundials: Suite of nonlinear and differential/algebraic equation solvers," ACM Transactions on Mathematical Software, vol. 31, no. 3, pp. 363-396, 2005.

[14] M. Agarwal, K. K. Park, R. N. Candler, M. A. Hopcroft, C. M. Jha, R. Melamud, B. Kim, B. Murmann, and T. W. Kenny, "Non-linearity cancellation in MEMS resonators for improved power-handling," in Proceedings of the IEEE International Electron Devices Meeting, 2005, pp. 295-298. 